Article

\title{
Processing Missile-Borne SAR Data by Using Cartesian Factorized Back Projection Algorithm Integrated with Data-Driven Motion Compensation
}

\author{
Min Bao ${ }^{1, *,+} \mathbb{D}$, Song Zhou ${ }^{2}$ and Mengdao Xing ${ }^{3,+}$ \\ 1 School of Electronic Engineering, Xidian University, Xi'an 710071, China \\ 2 School of Electronic Information and Engineering, Nanchang University, Nanchang 330031, China; \\ zhou_song@ncu.edu.cn \\ 3 National Laboratory of Radar Signal Processing, Xidian University, Xi'an 710071, China; xmd@xidian.edu.cn \\ * Correspondence: mbao@xidian.edu.cn; Tel.: +86-1357-291-4320 \\ + Current address: No. 2 South Taibai Road, Xi'an 710071, China.
}

Citation: Bao, M.; Zhou, S.; Xing, M. Processing Missile-Borne SAR Data by Using a Cartesian Factorized Back Projection Algorithm Integrated with Data-Driven Motion Compensation. Remote Sens. 2021, 13, 1462. https:// doi.org/10.3390/rs13081462

Academic Editor: Stefano Tebaldin

Received: 7 February 2021

Accepted: 7 April 2021

Published: 10 April 2021

Publisher's Note: MDPI stays neutra with regard to jurisdictional claims in published maps and institutional affiliations.

Copyright: (c) 2021 by the authors. Licensee MDPI, Basel, Switzerland. This article is an open access article distributed under the terms and conditions of the Creative Commons Attribution (CC BY) license (https:// creativecommons.org/licenses/by/ $4.0 /)$.

\begin{abstract}
Due to the independence of azimuth-invariant assumption of an echo signal, time-domain algorithms have significant performance advantages for missile-borne synthetic aperture radar (SAR) focusing with curve moving trajectory. The Cartesian factorized back projection (CFBP) algorithm is a newly proposed fast time-domain implementation which can avoid massive interpolations to improve the computational efficiency. However, it is difficult to combine effective and efficient data-driven motion compensation (MOCO) for achieving high focusing performance. In this paper, a new data-driven MOCO algorithm is developed under the CFBP framework to deal with the motion error problem for missile-borne SAR application. In the algorithm, spectrum compression is implemented after a CFBP process, and the SAR images are transformed into the spectrumcompressed domain. Then, the analytical image spectrum is obtained by utilizing wavenumber decomposition based on which the property of motion induced error is carefully investigated. With the analytical image spectrum, it is revealed that the echoes from different scattering points are aligned in the same spectrum range and the phase error becomes a spatial invariant component after spectrum compression. Based on the spectrum-compressed domain, an effective and efficient data-driven MOCO algorithm is accordingly developed for accurate error estimation and compensation. Both simulations of missile-borne SAR and raw data experiment from maneuvering highly-squint airborne SAR are provided and analyzed, which show high focusing performance of the proposed algorithm.
\end{abstract}

Keywords: analytical image spectrum; wavenumber; cartesian factorized back projection (CFBP); motion compensation (MOCO); missile-borne synthetic aperture radar

\section{Introduction}

Synthetic aperture radar (SAR) plays a significant role for microwave remote and sensing due to the ability of working all weather and all day and night [1-4]. As the hardware technology develops rapidly, SAR sensors can be compacted and mounted on highly maneuvering platforms [5,6], such as missile platforms [7-9]. SAR sensors on the missile platform can be used to acquire ground images for scene-matching guidance or obtain target images for precise bombing. Therefore, missile-borne SAR shows great potential in military application and has attracted growing interest in recent years.

The imaging algorithms for monstatic SAR focusing can be divided into two main categories: frequency-domain algorithms (FDAs) and time-domain algorithms (TDAs). FDAs include the range-Doppler algorithm (RDA), chirp-scaling algorithm (CSA), Omega-K algorithm, etc. These FDAs have been applied in many SAR systems with high computational efficiency [10,11]. FDAs are mostly based on the signal property of azimuth-invariant assumption. However, due to the geometry configuration and curve flying trajectory of 
missile platform, the azimuth-invariant assumption of missile borne SAR echoes is no longer valid and the range-azimuth coupling problem becomes much more difficult in the FDA process. Therefore, this paper concentrates on the TDA development for missile-borne SAR to obtain higher focusing performance.

TDAs are a significant trend for a monostatic SAR process [12-14], which is independent of azimuth-invariant assumption and has significant focusing performance advantages for missile-borne SAR focusing with curve moving trajectory. Back-projection (BP) is a widely used TDA $[15,16]$, which has high-accuracy focusing performance and has been well applied in many practical systems $[17,18]$. For SAR sensor operation on a missile platform, it is very necessary to develop a real-time processor for practical use. However, the huge computational burden of the BP algorithm prevents its advantages in real-time processes. Therefore, massive efforts have been devoted to accelerate the BP processing. A fast factorized BP (FFBP) algorithm and its modifications are one type of popular fast TDA [19-21]. It can reduce the computation burden by fusing the coarse angular resolution images generated from sub aperture (SA) echoes through image-domain interpolation [22,23]. However, the massive interpolation will bring a large computational burden. Cartesian factorized BP (CFBP) is a newly proposed fast TDA [24-26]. In CFBP, spectrum compression on SA image is used to decrease the Nyquist sampling rate (NSR) along the azimuth direction for high efficiency [27]. SA images are fused by azimuth upsampling and coherently processed in the Cartesian coordinate instead of pixel-by-pixel interpolation while the focusing accuracy can be comparable with the FFBP algorithm. CFBP has shown great potential on efficiency and accuracy improvement for SAR with arbitrary geometry configuration, especially for missile-borne SAR application.

However, to apply CFBP in missile-borne SAR process, it remains challenging to integrate with data-driven MOCO. Notably, it is practically significant to obtain well focused SAR images under motion error-contaminated systems [28-30]. In [17,31], an approximate Fourier transformation (FT) relationship has been revealed when the echoes are projected onto the polar coordinate under the FFBP scheme, based on which data-driven MOCO algorithm is proposed to be integrated with FFBP and achieve well focused results in airborne SAR and unmanned aerial vehicle (UAV) SAR applications. However, the FT relations are much more complicated in CFBP which will bring difficulty for MOCO development. In [32], a novel optimization-based MOCO is proposed and incorporated with fast TDA process which can obtain bistatic forward-looking SAR image with high accuracy and efficiency. However, in the case of intense motion error of missile platforms, the searching procedures may increase the computational burden obviously. In [27], an effective autofocusing algorithm with CFBP is proposed for spotlight SAR application and well focused images are obtained. However, it cannot achieve very high performance in missile-borne SAR application due to the curve platform trajectory and the complex phase error in echoes. Therefore, it remains a challenging to develop an efficient and effective data-driven MOCO algorithm with CFBP for missile-borne SAR applications.

In this paper, a new data-driven MOCO is developed under the CFBP scheme to address the motion error problem for missile-borne SAR focusing. After the CFBP process, spectrum compression is applied to the SAR image and the image is transformed into a new spectrum domain, which is referred to as a spectrum-compressed domain. In particular, the analytical image spectrum is derived by introducing wavenumber decomposition, based on which the property of motion induced error is carefully investigated. With the analytical image spectrum, it is revealed that the echoes from different scattering points are aligned in the same spectrum range and the phase error becomes spatial-invariant. With this inherent characteristic of spectrum-compressed domain, an effective and efficient data-driven MOCO algorithm is accordingly developed for accurate error estimation and compensation. Both simulation of missile-borne SAR and the raw data experiment from maneuvering highly-squint airborne SAR are utilized to evaluate the proposed algorithm, and the experimental results show its performance superiority in applications. 
This paper is organized as follows: the geometry and signal model of missile-borne SAR are introduced in Section 2. Section 3 derives the analytical image spectrum by introducing wavenumber decomposition. In Section 4, the implementation of spectrum compression is introduced and the property of motion induced error is carefully analyzed in spectrum-compressed domain. Section 5 develops the data-driven MOCO with CFBP and the processing procedures are discussed in detail. Results from both simulation and raw data experiments are provided and analyzed in Section 6. Finally, the conclusions are drawn in Section 7.

\section{Geometry and Signal Model}

The geometry is shown in Figure 1. To simplify the following derivations, only a twodimensional geometry model is assumed and considered. In practical application, the threedimensional geometry needs to be considered and projected onto the two-dimensional model for process [12]. In Figure 1, the SAR sensor is mounted on a missile-borne platform with a diving movement and continuously illuminating the interested area that has been highlighted in gray. Let us first consider the ideal case without trajectory deviation and assume that the platform is moving along the predetermined path, as denoted by the curve of $C$. Let $t$ denote the azimuth time. Assume that, at $t=0$, the antenna phase center (APC) of the radar is at $O$. To facilitate the CFBP process for achieving higher efficiency, we denote that the direction of the $y$-axis is along the beam direction at $t=0$ [24]. Thus far, the coordinate system of XOY is established. At $t$, the antenna phase center (APC) of the radar is at $P_{t}\left(x_{t}, y_{t}\right)$, where $x_{t}$ and $y_{t}$ can be regarded as a function of $t$, as

$$
\left\{\begin{array}{l}
x_{t}=f_{x}(t) \\
y_{t}=f_{y}(t)
\end{array} .\right.
$$

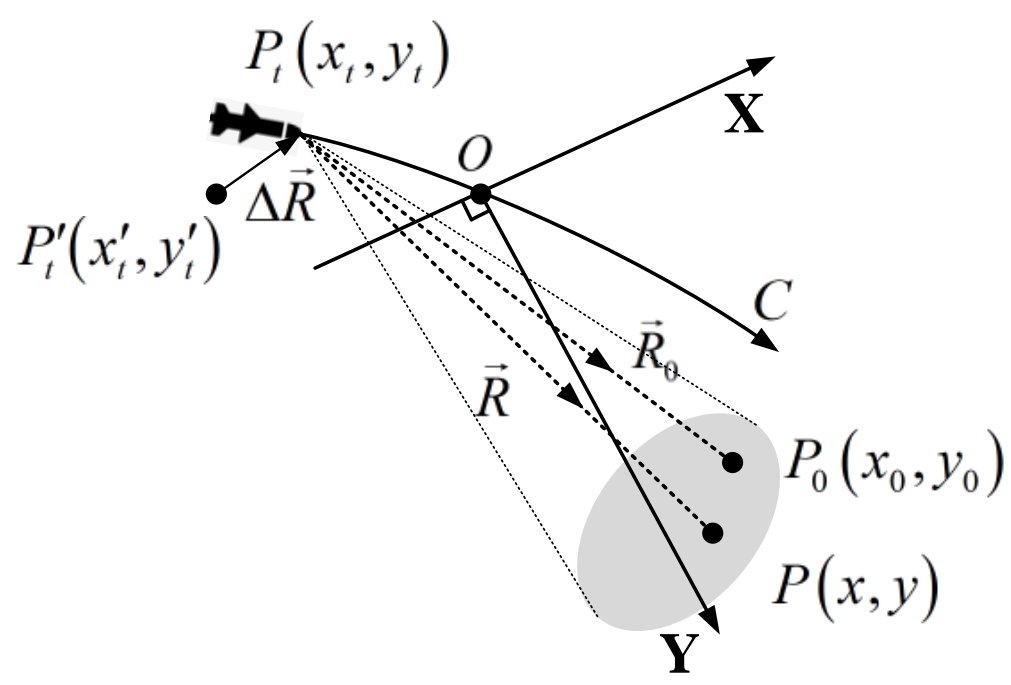

Figure 1. Missile-borne SAR geometry.

Consider an arbitrary illuminated scattterer of $P_{0}\left(x_{0}, y_{0}\right)$ with a constant reflectivity coefficient of 1 . At $t$, the echoes from $P_{0}$ can be given as

$$
S\left(x_{t}, K_{r}\right)=\exp \left(-j \vec{R}_{0} \cdot \vec{K}_{r}\right),
$$


where "." denotes the inner product operation, and $\vec{K}_{r}$ is the wavenumber vector of the echoes with the direction from $P_{t}$ to $P_{0}$ and the magnitude of $K_{r} . \overrightarrow{R_{0}}$ is the range vector from $P_{t}$ to $P_{0}$, which has the same direction of $\overrightarrow{K_{r}}$, and its length of $R_{0}$ is given by

$$
R_{0}=\sqrt{\left(x_{0}-x_{t}\right)^{2}+\left(y_{0}-y_{t}\right)^{2}} .
$$

In the direct BP algorithm, the echoes are projected onto a Cartesian grid of $P(x, y)$ to form an SAR image. The grid of $P(x, y)$ in the formed image is shown in Figure 1 , and the image obtained by $\mathrm{BP}$ algorithm can be given by the following integral [13]

$$
i(x, y)=\left.\int s\left(x_{t}, R\right)\right|_{R=\sqrt{\left(x-x_{t}\right)^{2}+\left(y-y_{t}\right)^{2}}} d x_{t},
$$

where

$$
s\left(x_{t}, R\right)=\int S\left(x_{t}, K_{r}\right) \exp \left(j R K_{r}\right) d K_{r} .
$$

By substituting (5) into (4), we obtain

$$
i(x, y)=\iint \exp \left[-j \sqrt{\left(x_{0}-x_{t}\right)^{2}+\left(y_{0}-y_{t}\right)^{2}} K_{r}+j \sqrt{\left(x-x_{t}\right)^{2}+\left(y-y_{t}\right)^{2}} K_{r}\right] d K_{r} d x_{t} .
$$

In (6), $y_{t}$ is the predetermined APC in a $y$-direction and $y_{t}=y\left(x_{t}\right)$ can be considered as a function with a variable $x_{t}$. According to the SAR geometry, (6) can be rewritten into the following form, as

$$
i(x, y)=\iint \exp \left(-j \overrightarrow{R_{0}} \cdot \overrightarrow{K_{r}}\right) \exp \left(j \vec{R} \cdot \overrightarrow{K_{r}}\right) d K_{r} d x_{t}
$$

where $\vec{R}$ is the range vector in the direction from $P_{t}$ to the grid $P(x, y)$. The length of $\vec{R}$ can be given by

$$
R=\sqrt{\left(x-x_{t}\right)^{2}+\left(y-y_{t}\right)^{2}} .
$$

Then, we consider the practical case with trajectory deviation. Assuming that, at $t$, the APC of SAR sensor is at $P_{t}^{\prime}\left(x_{t}^{\prime}, y_{t}^{\prime}\right)$ which is deviated from $P_{t}\left(x_{t}, y_{t}\right)$, as shown in Figure 1. Let $\Delta \vec{R}$ denote the vector of the trajectory deviation at $t$, and $\Delta \vec{R}$ can be also regarded as a function of $x_{t}$ as

$$
\Delta \vec{R}=\Delta \overrightarrow{R\left(x_{t}\right)} .
$$

With $\Delta \vec{R},(7)$ can be rewritten as

$$
i(x, y)=\iint \exp \left[-j\left(\overrightarrow{R_{0}}+\Delta \vec{R}\right) \cdot \overrightarrow{K_{r}^{\prime}}\right] \exp \left(j \vec{R} \cdot \overrightarrow{K_{r}}\right) \mathrm{d} K_{r} \mathrm{~d} x_{t}
$$

where $\overrightarrow{K_{r}^{\prime}}$ is the wavenumber vector with the direction from $P_{t}^{\prime}$ to $P_{0}$. Since the magnitude of $\Delta \vec{R}$ is generally much smaller than that of $\overrightarrow{R_{0}}, \overrightarrow{K_{r}^{\prime}}$ can be approximated by $\overrightarrow{K_{r}}$ in (10) to obtain

$$
i(x, y) \approx \iint \exp \left[-j\left(\overrightarrow{R_{0}}+\Delta \vec{R}\right) \cdot \overrightarrow{K_{r}}\right] \exp \left(j \vec{R} \cdot \overrightarrow{K_{r}}\right) d K_{r} d x_{t}
$$

By comparing (11) with (7), it can be noted that the deviation term of $\Delta \vec{R}$ is introduced into the integral. The deviation of $\Delta \vec{R}$ can be also be referred to as motion error [33], which 
needs to be specifically considered for missile-borne SAR focusing. In the following sections, the analytical image spectrum of CFBP will be investigated carefully and an effective MOCO strategy will be developed with the CFBP process to move the influence of $\Delta \vec{R}$.

\section{Spectrum Analysis}

Before developing effective data-driven MOCO algorithm, it is necessary to analyze the property of motion induced error in image spectrum domain since classic autofocusing techniques, e.g., phase gradient autofocusing (PGA) [34] and map-drift autofocusing (MDA) [28], are mostly based on the phase history domain for error estimation and compensation. To derive the analytical form of the image in a Cartesian coordinate system, a pair of orthogonal wavenumber vectors, $\overrightarrow{K_{x}}$ and $\overrightarrow{K_{y}}$, are introduced, where $\overrightarrow{K_{x}}$ is in the $x$-direction and $\overrightarrow{K_{y}}$ is in the $y$-direction, as shown in Figure 2. Then, the wavenumber in (11) can be decomposed as [31]

$$
\overrightarrow{K_{r}}=\overrightarrow{K_{x}}+\overrightarrow{K_{y}}
$$

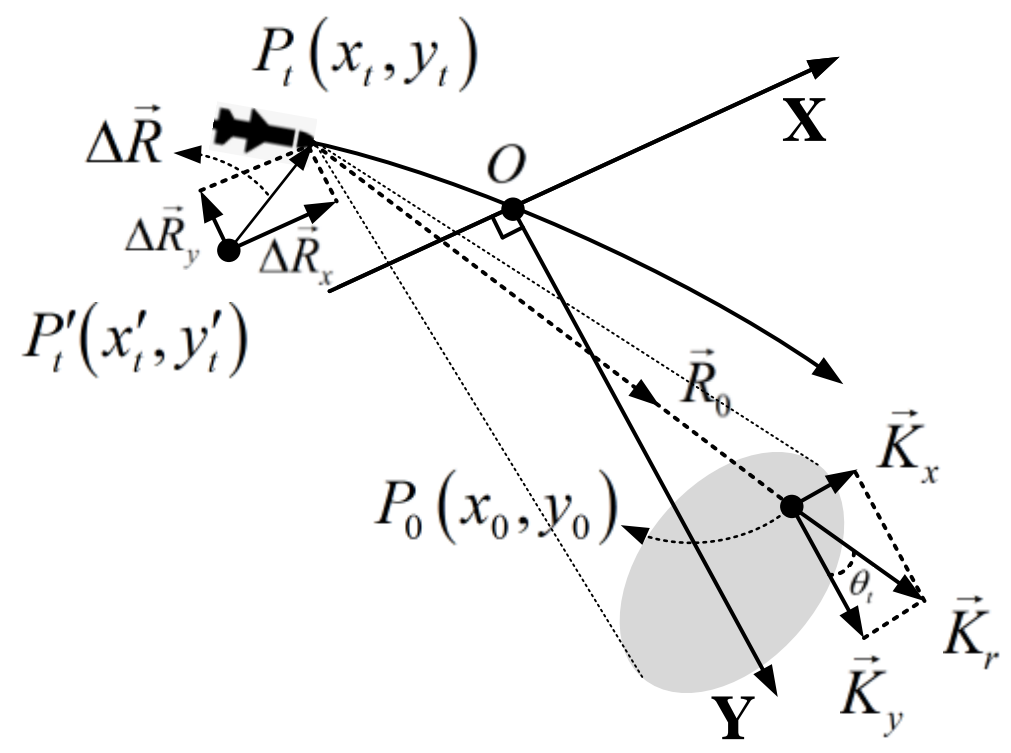

Figure 2. Wavenumber decomposition.

The range vectors as well as motion error of $\Delta \vec{R}$ in (11) can be also decomposed along $x$ - and $y$-directions as

$$
\left\{\begin{array}{l}
\overrightarrow{R_{0}}=\overrightarrow{x_{0}}+\overrightarrow{y_{0}} \\
\vec{R}=\vec{x}+\vec{y} \\
\Delta \vec{R}=\Delta \overrightarrow{R_{x}}+\Delta \overrightarrow{R_{y}}
\end{array}\right.
$$

By substituting (12) and (13) into (11), after manipulation, it obtains

$$
\begin{aligned}
i(x, y) \approx \int & \int \exp \left(-j \Delta R_{x} K_{x}-j \Delta R_{y} K_{y}\right) \\
& \times \exp \left(-j x_{0} K_{x}-j y_{0} K_{y}\right) \exp \left(j x K_{x}+j y K_{y}\right) d K_{x} d K_{y},
\end{aligned}
$$

where $\Delta R_{x}$ and $\Delta R_{y}$ are the magnitudes of $\overrightarrow{R_{x}}$ and $\overrightarrow{R_{y}}$, respectively. $K_{x}$ and $K_{y}$ are the magnitudes of $\overrightarrow{K_{x}}$ and $\overrightarrow{K_{y}}$, respectively. For obtaining (14), a 2D integral transformation is 
applied to (11). In the SAR algorithm development, we mainly concern the phase modulation rather than magnitude modulation, so that the Jacobian determinant introduced by the 2D integral transformation is ignored [31]. The detailed derivation of (14) can be found in [31]. The first exponential term in (14) is the phase error term introduced by $\Delta \vec{R}$, which should be compensated in the MOCO. With (14), the analytical image spectrum can be obtained as

$$
I\left(K_{x}, K_{y}\right) \approx \exp \left(-j \Delta R_{x} K_{x}-j \Delta R_{y} K_{y}\right) \exp \left(-j x_{0} K_{x}-j y_{0} K_{y}\right) .
$$

Equation (15) denotes the analytical image spectrum with one single scatterer of $P_{0}$. The range of $K_{x}$ is also corresponding to the resolution in the $x$-direction. Then, we consider the range of $K_{x}$. Let $\theta_{t}$ denote the angle between $\overrightarrow{K_{r}}$ and $\overrightarrow{K_{y}}$, as shown in Figure 2, and it has

$$
\sin \theta_{t}=\frac{\left(x_{0}-x_{t}\right)}{\sqrt{\left(x_{0}-x_{t}\right)^{2}+\left(y_{0}-y_{t}\right)^{2}}},
$$

and the relationship between $K_{x}$ and $K_{r}$ can be approximated by $K_{x} \approx K_{r c} \sin \theta_{t}$, as

$$
K_{x} \approx \frac{K_{r c}\left(x_{0}-x_{t}\right)}{\sqrt{\left(x_{0}-x_{t}\right)^{2}+\left(y_{0}-y_{t}\right)^{2}}},
$$

where $K_{r c}$ is the center of $K_{r}$. For most missile-borne SAR applications with media resolution in azimuth, e.g., lower than $1 \mathrm{~m}$ resolution, the magnitudes of $x_{t}, x_{0}$ as well as $y_{t}$ are much smaller than the range of $y_{0}$. Thus, (17) can be further approximated by

$$
K_{x} \approx \frac{K_{r c}\left(x_{0}-x_{t}\right)}{y_{0}}
$$

From (18), it can be seen that the range of $K_{x}$ is highly dependent on the position coordinate of $x_{0}$. In other words, different point scatterers in the image spectrum domain exhibit different range center of $K_{x}$. Here, we consider a typical set of missile-borne SAR parameters given in Table 1 for example, and nine point scatterers in the illumination are simulated. The locations of the nine point scatterers are listed in Table 2 and shown in Figure 3. For better observing the image spectrum of different scatterers, the nine points are assumed to have a different range in the $y$-direction. The obtained image from CFBP algorithm is then transformed into phase history domain, i.e., $\left(K_{x}, y\right)$ domain, as shown in Figure 4. From Figure 4, it can be seen that the spectrum of different scatterers is spread into a different range in the $K_{x}$ direction. Because of the different spectrum range, the contained phase error in the spectrum domain becomes spatial variant which brings difficulty for accurate error estimation and compensation. In the following section, a new process strategy will be developed to deal with the spatial-variant problem based on which an effective data-driven $\mathrm{MOCO}$ will be proposed. 
Table 1. Parameters of a missile-borne SAR system.

\begin{tabular}{cc}
\hline Wave Band & $\mathbf{K u}$ \\
\hline Bandwidth & $180 \mathrm{MHz}$ \\
Range Center & about $10,000 \mathrm{~m}$ \\
Velocity in the $x$-Direction & $500 \mathrm{~m} / \mathrm{s}$ \\
Acceleration in $x$-Direction & $-15 \mathrm{~m} / \mathrm{s}^{2}$ \\
Velocity in $y$-Direction & $120 \mathrm{~m} / \mathrm{s}$ \\
Acceleration in $y$-Direction & $-12 \mathrm{~m} / \mathrm{s}^{2}$ \\
Synthetic Duration & $0.5 \mathrm{~s}$ \\
\hline
\end{tabular}

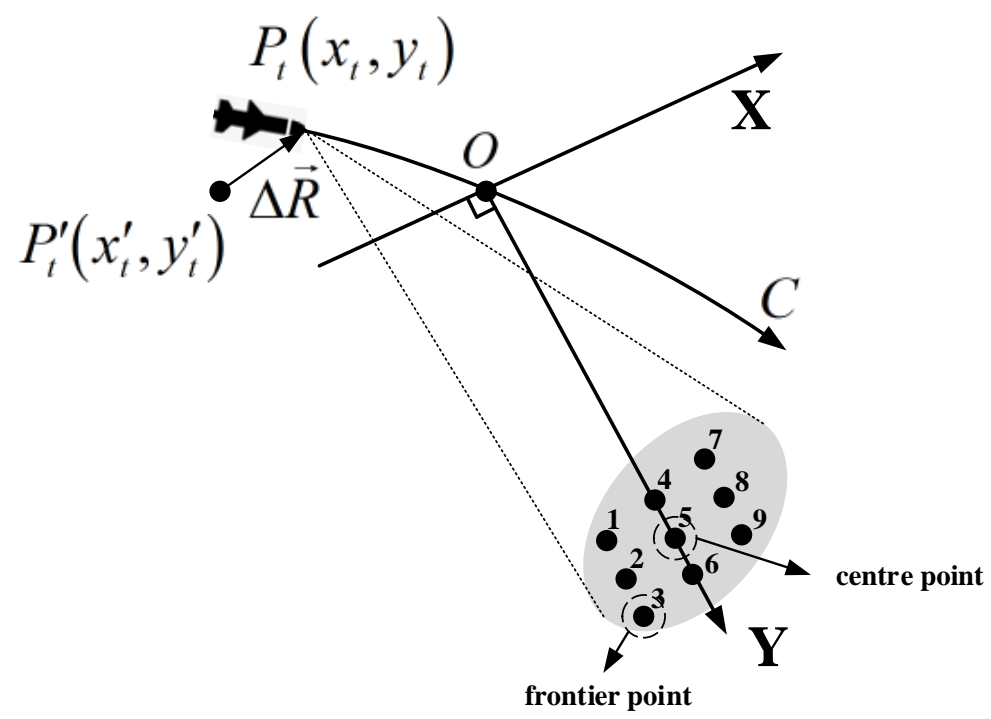

Figure 3. Geometry and point locations.

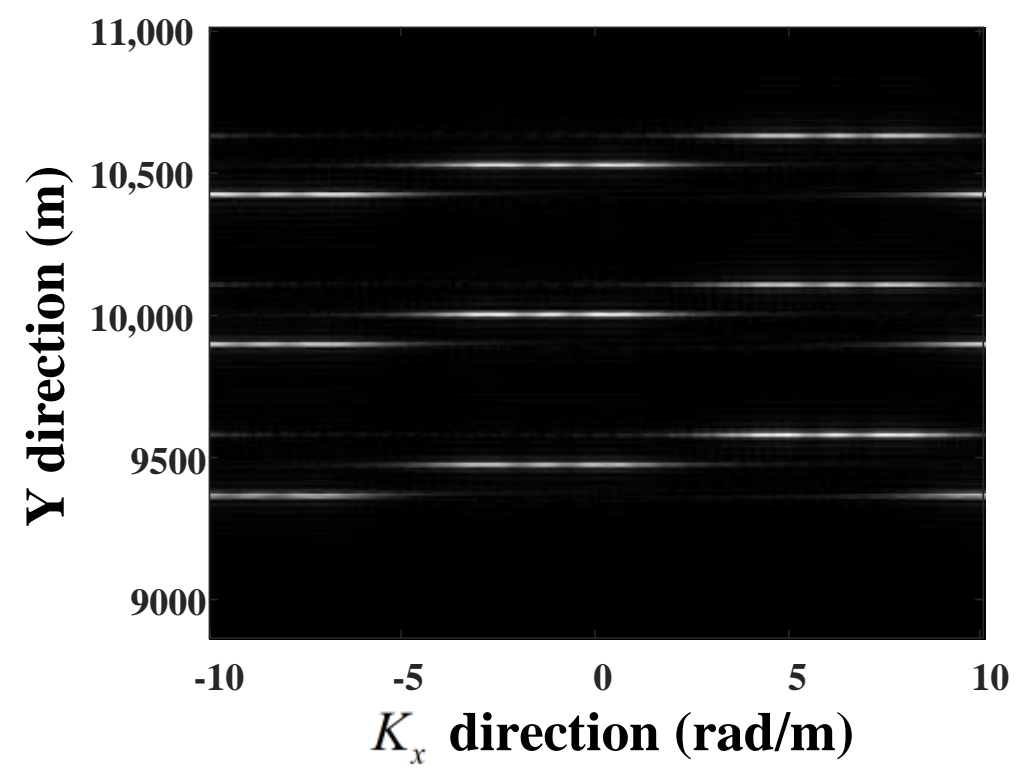

Figure 4. Image spectrum of nine point scatterers after the CFBP process. 
Table 2. The locations of the point scatterers.

\begin{tabular}{ccc}
\hline Point & X & Y \\
\hline Point 1 & $-500 \mathrm{~m}$ & $9600 \mathrm{~m}$ \\
Point 2 & $-500 \mathrm{~m}$ & $10,100 \mathrm{~m}$ \\
Point 3 & $-500 \mathrm{~m}$ & $10,600 \mathrm{~m}$ \\
Point 4 & $0 \mathrm{~m}$ & $9500 \mathrm{~m}$ \\
Point 5 & $0 \mathrm{~m}$ & $10,000 \mathrm{~m}$ \\
Point 6 & $0 \mathrm{~m}$ & $10,500 \mathrm{~m}$ \\
Point 7 & $500 \mathrm{~m}$ & $9400 \mathrm{~m}$ \\
Point 8 & $500 \mathrm{~m}$ & $9900 \mathrm{~m}$ \\
Point 9 & $500 \mathrm{~m}$ & $10,400 \mathrm{~m}$ \\
\hline
\end{tabular}

\section{Data-Driven MOCO in the Spectrum-Compressed Domain}

To address the problem of spreading image spectrum and the spatial-variant phase error, an implementation of spectrum compression is introduced into the CFBP process. The function for spectrum compression is given as

$$
F_{s c}(x, y)=\exp \left(-j K_{r c} \sqrt{x^{2}+y^{2}}\right)
$$

where $K_{r c}$ is the center of $K_{r}$ described in (10). $x$ and $y$ denote the coordinates of the grid in the formed image. Actually, the spectrum compression of (19) is also an inherit stage in the CFBP process [24]; however, we particularly analyze the spectrum compression to show its advantages which will facilitate the MOCO development. Here, we introduce a wavenumber of $\overrightarrow{K_{s c}}$ and a range vector of $\vec{r}$, both of which are with the direction from the origin of $O$ to the grid of $(x, y)$. Note that the direction of $\overrightarrow{K_{s c}}$ is different from that of $\overrightarrow{K_{r}}$ introduced in (2). In (2), the direction of $\vec{K}_{r}$ is from $P_{t}$ to $P_{0}$. The magnitude of $\overrightarrow{K_{s c}}$ is $K_{r c}$, while the magnitude of $\vec{r}$ can be given as

$$
r=\sqrt{x^{2}+y^{2}}
$$

(19) and (20) are rewritten as

$$
F_{s c}(x, y)=\exp \left(-j \overrightarrow{K_{s c}} \vec{r}\right)
$$

Then, both $\overrightarrow{K_{s c}}$ and $\vec{r}$ are decomposed along $x$ - and $y$-directions, as

$$
\left\{\begin{array}{l}
\overrightarrow{K_{s c}}=\overrightarrow{K_{x c}}+\overrightarrow{K_{y c}} \\
\vec{r}=\vec{x}+\vec{y}
\end{array} .\right.
$$

By substituting (22) into (21), it has

$$
F_{s c}(x, y)=\exp \left(-j x K_{x c}-j y K_{y c}\right)
$$

where $K_{x c}$ and $K_{y c}$ are the magnitudes of $\overrightarrow{K_{x c}}$ and $\overrightarrow{K_{y c}}$, respectively. By multiplying (14) with (23), it obtains

$$
\begin{aligned}
\widetilde{i}(x, y) \approx \int & \int \exp \left(-j \Delta R_{x} K_{x}-j \Delta R_{y} K_{y}\right) \\
& \times \exp \left(-j x_{0} K_{x}-j y_{0} K_{y}\right) \exp \left(j x K_{x}+j y K_{y}\right) \exp \left(-j x K_{x c}-j y K_{y c}\right) d K_{x} d K_{y},
\end{aligned}
$$


In (24), $\overrightarrow{K_{x c}}$ is the decomposition of $\overrightarrow{K_{s c}}$ in the $x$-direction. According to the geometry of Figure 2 and similarly to the analyses of (18), the relationship between $\overrightarrow{K_{x c}}$ and $\overrightarrow{K_{s c}}$ can be given by

$$
K_{x c} \approx \frac{K_{r c} x_{0}}{y_{0}}
$$

Note, in (24) that $K_{x c}$ and $K_{y c}$ are exactly the center of $K_{y}$ and $K_{y}$ for an arbitrary grid of $(x, y)$. Let

$$
\left\{\begin{array}{l}
K_{x}=K_{x c}+\Delta K_{x} \\
K_{y}=K_{y c}+\Delta K_{y}
\end{array}\right.
$$

Then, by substituting (26) into (27), it obtains

$$
\begin{aligned}
\widetilde{i}(x, y) \approx \iint & \exp \left(-j x_{0} K_{x c}-j y_{0} K_{y c}\right) \\
& \times \exp \left(-j \Delta R_{x} K_{x c}-j \Delta R_{x} \Delta K_{x}-j \Delta R_{y} K_{y c}-j \Delta R_{y} \Delta K_{y}\right) \\
& \times \exp \left(-j x_{0} \Delta K_{x}-j y_{0} \Delta K_{y}\right) \exp \left(j x \Delta K_{x}+j y \Delta K_{y}\right) \Delta K_{x} d \Delta K_{y},
\end{aligned}
$$

and the analytical image spectrum becomes

$$
\begin{aligned}
\widetilde{I}\left(\Delta K_{x}, \Delta K_{y}\right) & \approx \exp \left(-j x_{0} K_{x c}-j y_{0} K_{y c}\right) \\
& \times \exp \left(-j \Delta R_{x} K_{x c}-j \Delta R_{x} \Delta K_{x}-j \Delta R_{y} K_{y c}-j \Delta R_{y} \Delta K_{y}\right) \\
& \times \exp \left(-j x_{0} \Delta K_{x}-j y_{0} \Delta K_{y}\right) .
\end{aligned}
$$

Thus far, the analytical image spectrum after spectrum compression is obtained, and it can be noted that the image has been transformed into a spectrum-compression domain. In practical application, we utilize (19) instead of (23) for spectrum compression. In (28), the first exponential term is a constant which is introduced by the spectrum compression and will not bring defocusing to the final image. The second exponential term is the phase error term introduced by the motion error. In the data-driven MOCO, the high-order phase error components need to be estimated and compensated for achieving high focusing quality. Finally, we consider the last exponential term in (28), as well as the range of $\Delta K_{x}$. According to (18), (25) and (26), the range of $\Delta K_{x}$ can be given by

$$
\Delta K_{x} \approx \frac{-K_{r c} x_{t}}{y_{0}} .
$$

From (29), it can be seen that the new wavenumber of $\Delta K_{x}$ in the last exponential of (28) is no longer dependent on the coordinate of $x_{0}$ of scatterer. In other words, the image spectrum of all the scatterers will be aligned in the same spectrum range. Figure 5 presents the image spectrum in the range history domain of $\left(K_{x}, y\right)$ after spectrum compression. The SAR geometry as well as all the radar parameters are according to Figure 3 and Table 1. From Figure 5, it can be seen that the image spectrum of different scatterers exhibits nearly the same spectrum range and the phase error contained in the echoes can be regarded as spatial-invariant components that will significantly facilitate data-driven MOCO development. 


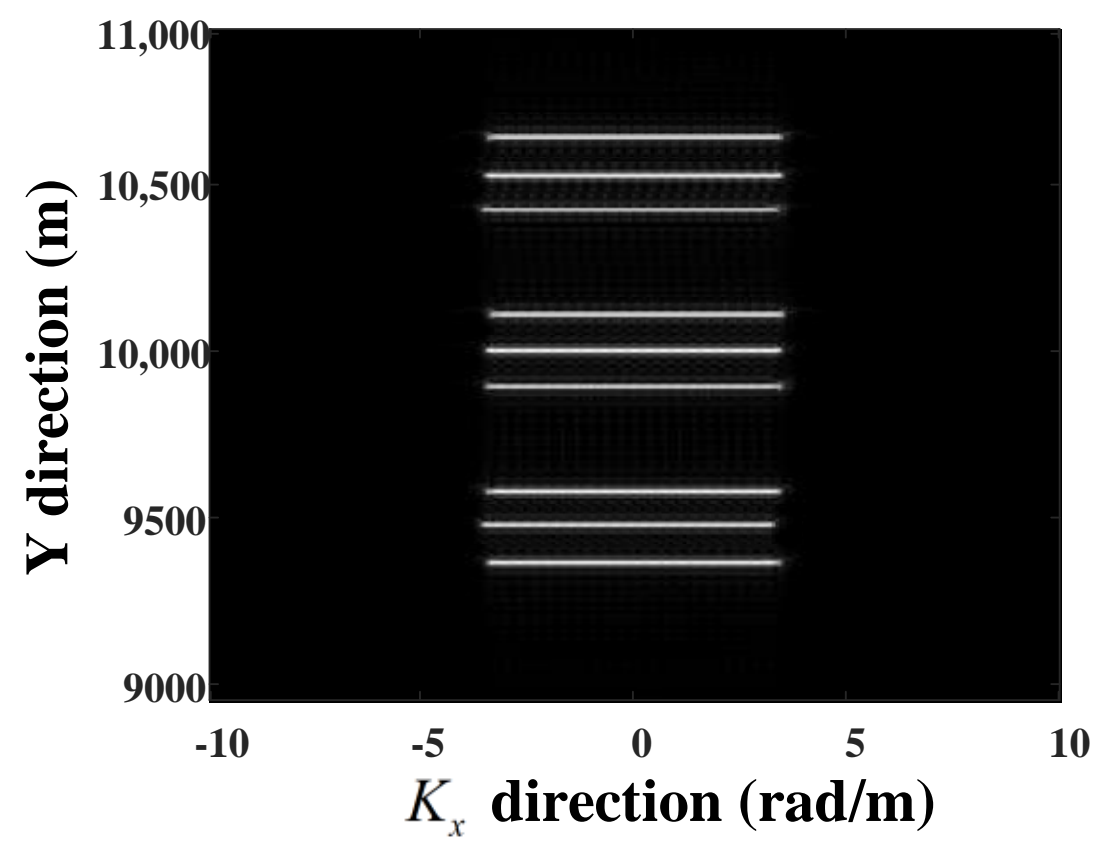

Figure 5. Image spectrum of nine point scatterers in a spectrum-compressed domain.

It is also emphasized that the approximation from (17) to (18) is based on the fact that the motion of the missile-platform is much smaller than the range of $R$. Generally for most missile-borne SAR applications with media resolution, e.g., lower than $1 \mathrm{~m}$ resolution in azimuth, the approximation is accurate enough for the MOCO development.

\section{Processing Procedures}

The processing procedures of data-driven MOCO combined with CFBP is summarized in Figure 6, which includes three main steps.

Step 1: CFBP processing. In the first step, the raw echoed signal collected in missileborne SAR sensor will be preprocessed, e.g., range compressing or range deramp processing. Then, the pre-processed signal is processed by CFBP for imagery. The on-board inertial navigation system/global positioning system (INS/GPS) information is readily incorporated into CFBP for preliminary MOCO. If the INS/GPS is not accurate enough, the proposed $\mathrm{MOCO}$ algorithm is utilized to compensate for the unknown motion error in a data-driven manner. It is noted that the Nyquist sample rate in every recursion in CFBP needs to be high enough, so that the information of both echoed signal and motion error will be fully contained and transformed in the images in every recursion, and only the image obtained from final recursion is utilized for the MOCO process. After step 1, the coarsely focused image of $i(x, y)$ from CFBP is obtained.

Step 2: Spectrum compression and MOCO. In the second step, the image obtained from CFBP is transformed into a spectrum-compressed domain by utilizing spectrum compression according to (19). Then, the image is transformed into phase history domain, i.e., $\tilde{I}\left(K_{x}, y\right)$ domain, by applying fast Fourier transformation (FFT) along the $x$-direction. Because the echoes from different scatterers have nearly the same spectrum range along $K_{x}$ direction, classic weighted PGA (WPGA) [35] is processed with high efficiency and effectiveness. It is also noted that both azimuth phase error (APE) and nonsystematic range cell migration (NsRCM) need to be considered in the MOCO for high-accuracy estimation and compensation, and the compensated image of $\tilde{I}_{M O C O}\left(K_{x}, y\right)$ is obtained. The compensation for NsRCM can be referred to [31,36].

Step 3: After both APE and NsRCM are compensated in phase history domain, the images are transformed back into the $(x, y)$ domain by utilizing inverse FFT (IFFT) along the $K_{x}$ direction. Then, the image is transformed into $(x, y)$ domain and a spectrum- 
decompression is applied to the image to preserve the phase information. The spectrumdecompression function is a conjunction of spectrum-compression function, given as

$$
F_{s d c}(x, y)=\exp \left(j K_{r c} \sqrt{x^{2}+y^{2}}\right) .
$$

After spectrum-decompression, the image of $i_{M O C O}(x, y)$ with high focusing quality is finally obtained.

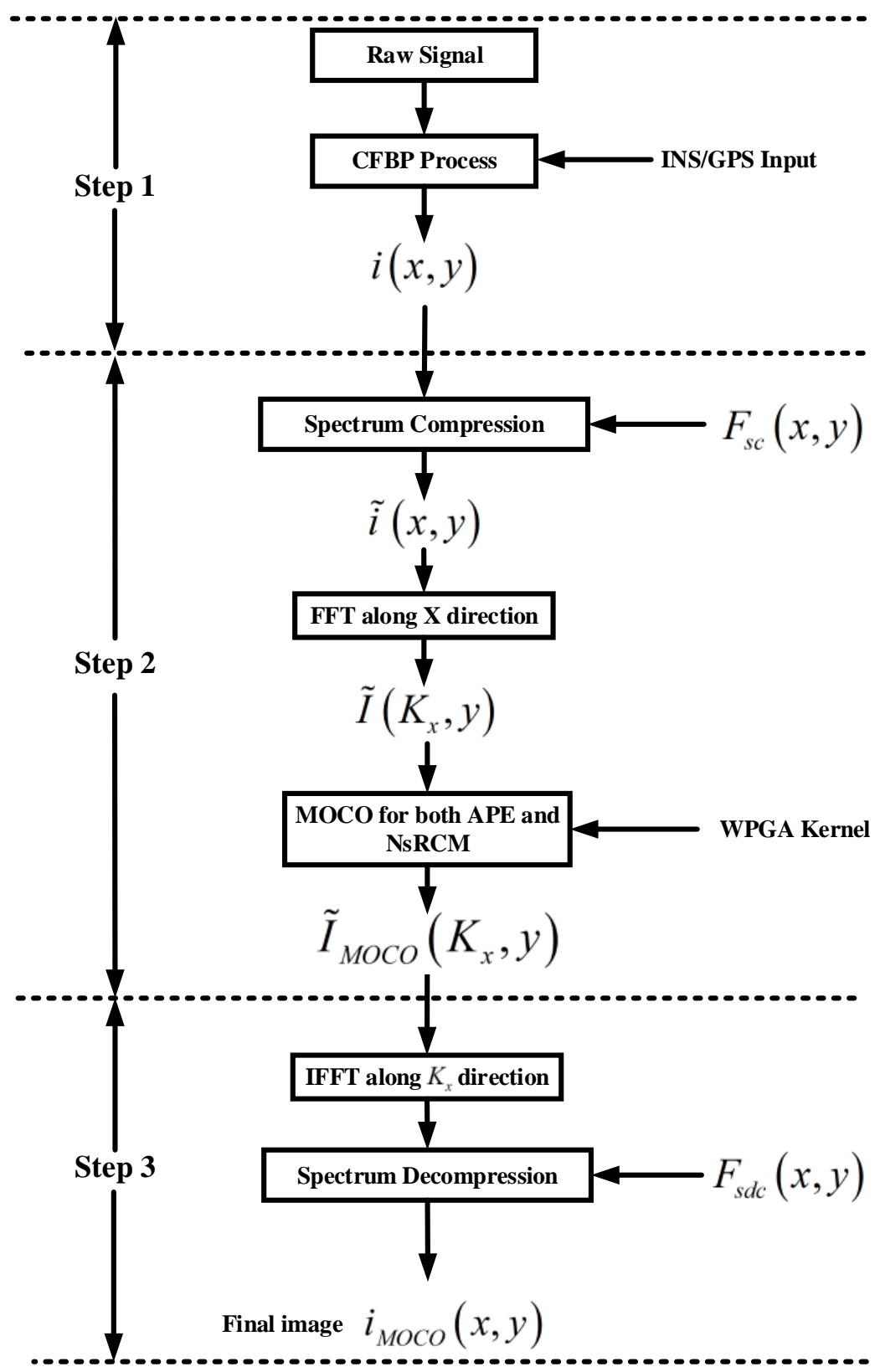

Figure 6. Processing procedures.

\section{Simulations and Raw Data Experiments}

In this section, both simulations for missile-borne SAR and raw data experiments of highlysquint airborne SAR are presented to evaluate the performance of the proposed algorithm. 


\subsection{Simulations for Missile-Borne SAR}

In this sub-section, simulation experiments are presented and analyzed to evaluate the performance of the data-driven MOCO. The missile-borne SAR geometry for the simulation is according to Figure 3, and nine point scatterers in the observing scene for analyzing is according to Table 2. The parameters are according to Table 1. Figure 7 shows the assumed motion errors of the missile platform in both $x$ - and $y$-directions.
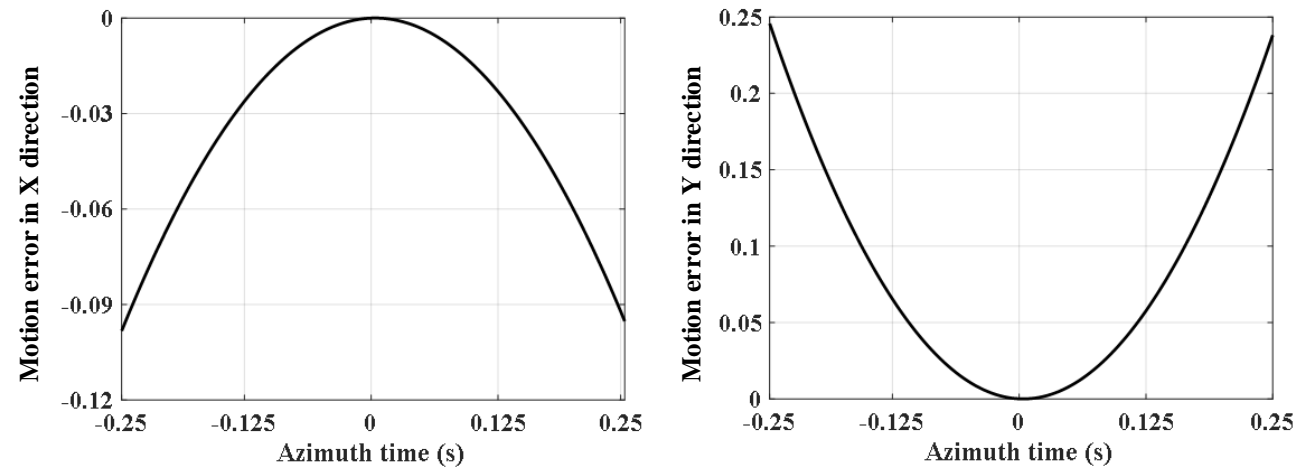

Figure 7. Assumed motion errors in $x$ - and $y$-directions.

The algorithms for the simulation experiments are programmed on a MATLAB platform and Windows 10 system on a desktop computer with an i7-9700 CPU and 32 GB RAM without parallel processing. The total processing time using direct BP is approximately $175 \mathrm{~min}$. The total processing time by using conventional FFBP is approximately $11.4 \mathrm{~min}$. The total processing time using the algorithm of [27] is about $4.1 \mathrm{~min}$. The total processing time by using the proposed MOCO combined with CFBP is approximately $2.7 \mathrm{~min}$. It can be seen that the proposed MOCO with CFBP is much faster than direct BP and FFBP algorithms, as well as the algorithm of [27]. In Figure 3, point 5 is regarded as the center point while point 3 is regarded as the frontier point, and the results of both center and frontier points are particularly analyzed. In the MOCO process, the phase error varying along range direction is also considered and compensated according to [29].

Figure 8 presents the focusing results of the center point and frontier point obtained from the CFBP algorithm without any MOCO. The results degraded seriously along the azimuth direction due to the unknown motion error, and the defocusing results bring difficulty to the subsequent process. Figure 9 presents the focusing results of the center point and frontier point obtained from CFBP algorithm in which the MOCO is processed without spectrum compression. From Figure 9, it can be seen that both center and frontier points still have some defocusing. Because the echoes from different point scatterers have a different spectrum range along the $K_{x}$ direction and the phase error is spatially variant, the phase error cannot be exactly estimated and compensated. Figure 10 presents the focusing results of the center point and frontier point obtained from the MOCO algorithm of [27]. Table 3 presents the $-3 \mathrm{~dB}$ main-lobe, the peak-sidelobe ration (PSLR) and the integrated sidelobe ratio (ISLR) for the focusing quality analyses of the nine point scatterers. Because the spatial-variant phase error cannot be accurately compensated, minor defocusing still remains in the final image. Figure 11 presents the focusing results of the center point and frontier point obtained from CFBP algorithm in which the MOCO is processed after spectrum compression. From Figure 11, it can be seen that the focusing performance of the proposed algorithm obtains high focusing performance, where the processing results have a narrower main-lobe and lower sidelobes. The $-3 \mathrm{~dB}$ main-lobe, the peak-sidelobe ration (PSLR) and the integrated sidelobe ratio (ISLR) for the focusing quality analyses of the nine point scatterers are listed in Table 4 . The high focusing quality demonstrates the superior performance of the proposed MOCO for missile-borne SAR application. 

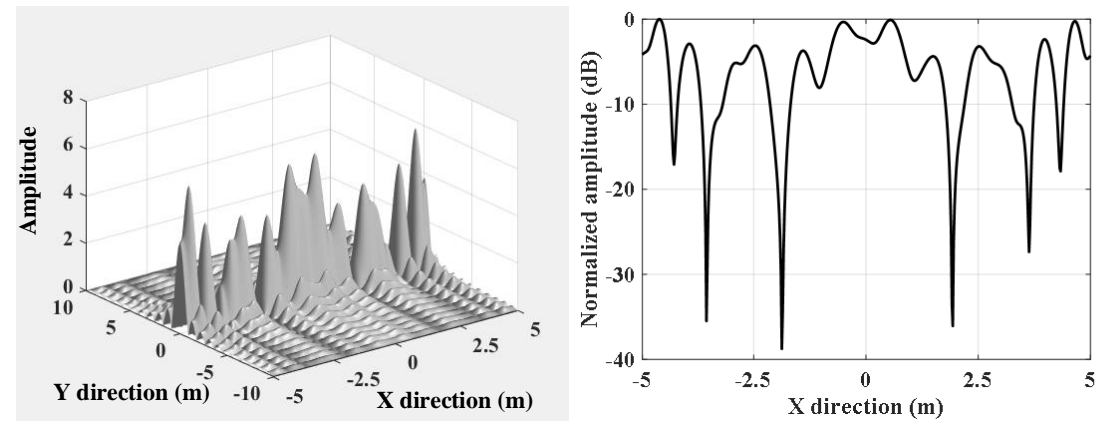

(a)
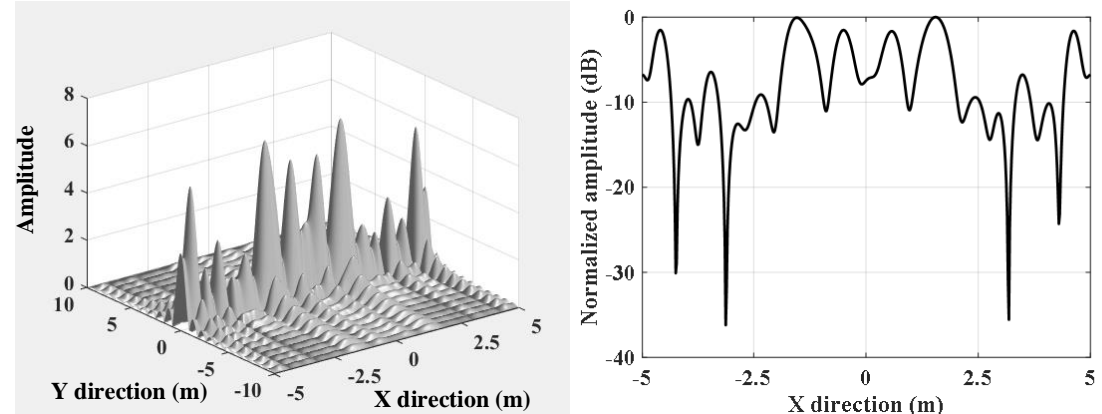

(b)

Figure 8. Results from CFBP without any MOCO. (a) Focusing results of center point and its azimuth responses; (b) focusing results of frontier point and its azimuth responses.
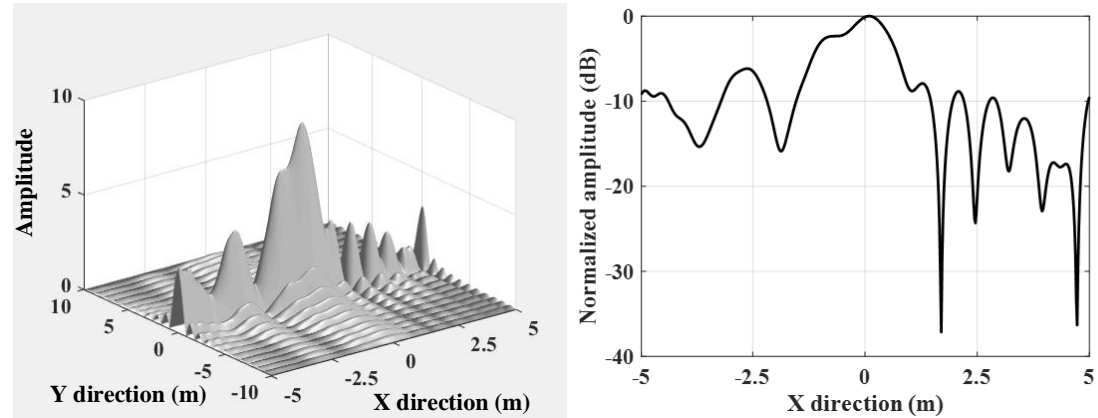

(a)
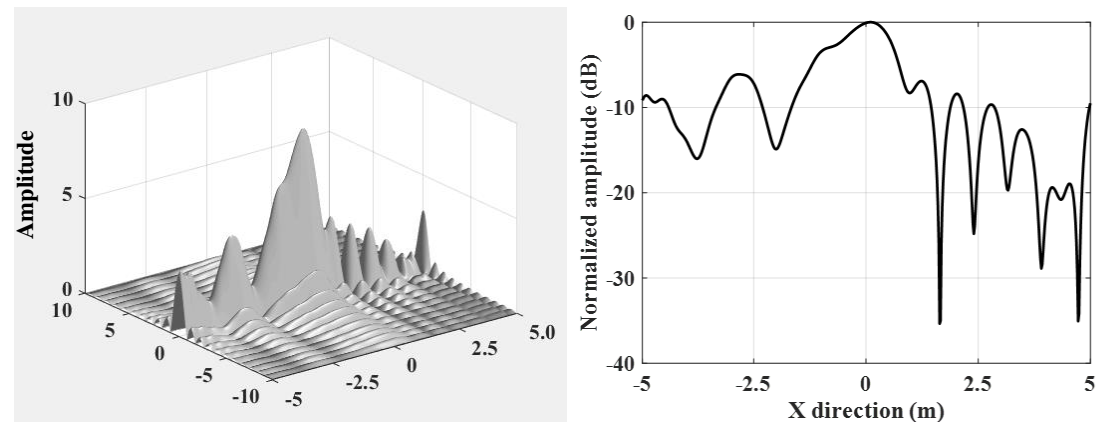

(b)

Figure 9. Results from $\mathrm{CFBP}$ and $\mathrm{MOCO}$ are processed without spectrum compression. (a) Focusing results of center point and its azimuth responses; (b) focusing results of frontier point and its azimuth responses. 

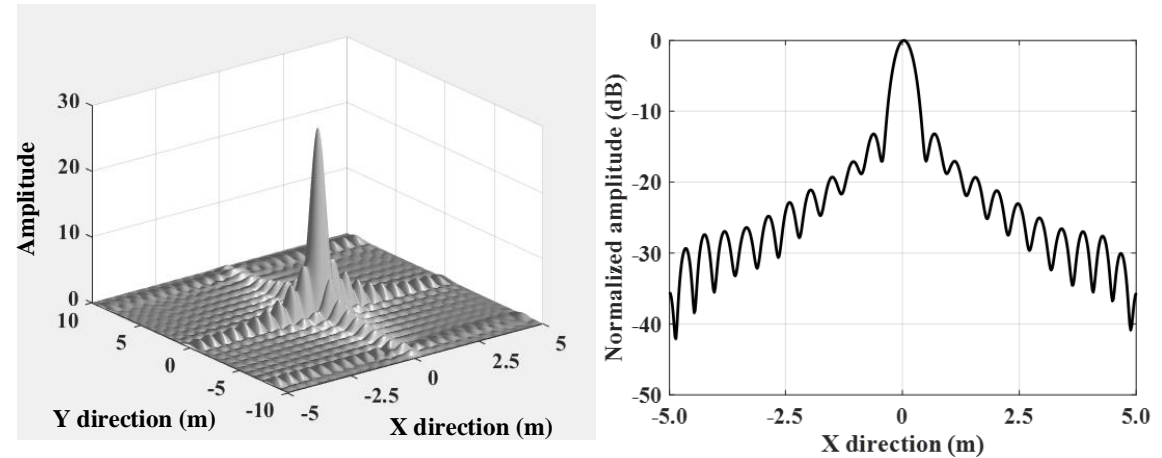

(a)
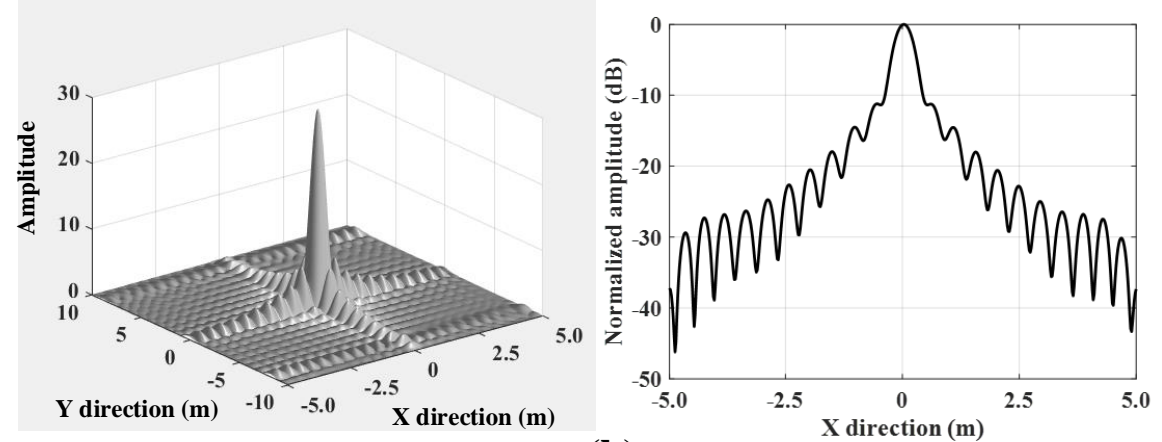

(b)

Figure 10. Results from the algorithm of reference [27]. (a) Focusing results of center point and its azimuth responses; (b) focusing results of frontier point and its azimuth responses.
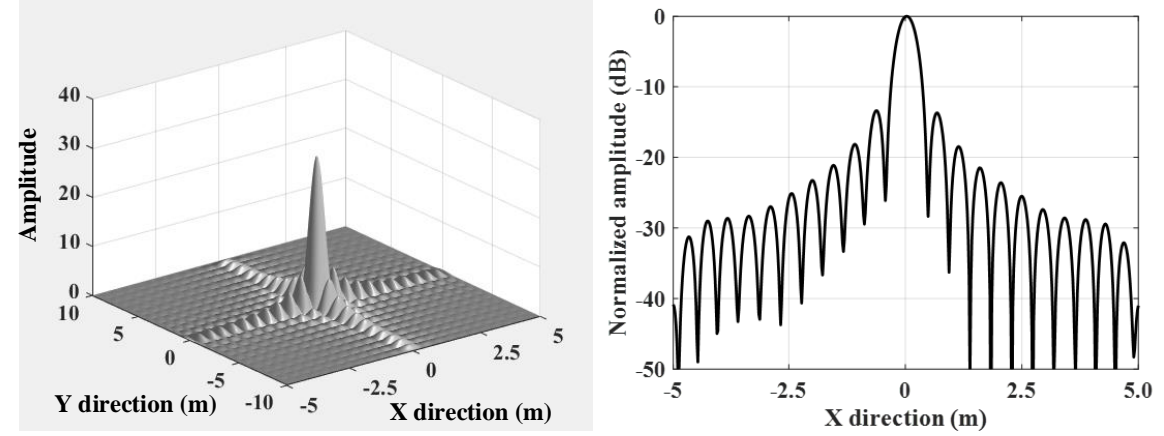

(a)
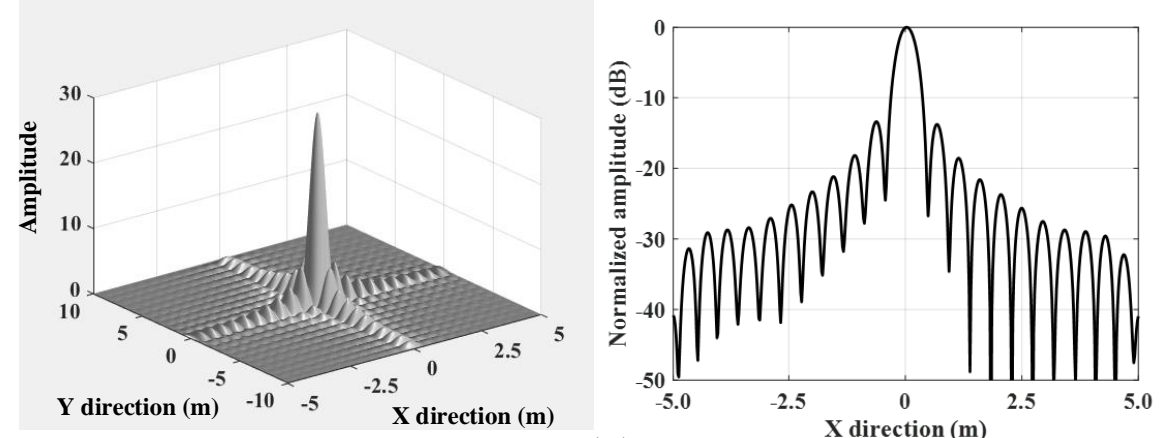

(b)

Figure 11. Results from the proposed algorithm. (a) Focusing results of center point and its azimuth responses; (b) focusing results of frontier point and its azimuth responses. 
Table 3. The focusing quality of the point scatterers from the algorithm of [27].

\begin{tabular}{|c|c|c|c|}
\hline \multicolumn{4}{|c|}{$y$-Direction } \\
\hline Point & 3-dB width & PSLR & ISLR \\
\hline Point 1 & $0.86 \mathrm{~m}$ & $-12.91 \mathrm{~dB}$ & $-9.98 \mathrm{~dB}$ \\
\hline Point 2 & $0.85 \mathrm{~m}$ & $-13.11 \mathrm{~dB}$ & $-9.91 \mathrm{~dB}$ \\
\hline Point 3 & $0.86 \mathrm{~m}$ & $-13.02 \mathrm{~dB}$ & $-9.84 \mathrm{~dB}$ \\
\hline Point 4 & $0.85 \mathrm{~m}$ & $-13.10 \mathrm{~dB}$ & $-9.88 \mathrm{~dB}$ \\
\hline Point 5 & $0.85 \mathrm{~m}$ & $-13.05 \mathrm{~dB}$ & $-9.89 \mathrm{~dB}$ \\
\hline Point 6 & $0.86 \mathrm{~m}$ & $-13.02 \mathrm{~dB}$ & $-9.91 \mathrm{~dB}$ \\
\hline Point 7 & $0.86 \mathrm{~m}$ & $-12.94 \mathrm{~dB}$ & $-9.77 \mathrm{~dB}$ \\
\hline Point 8 & $0.87 \mathrm{~m}$ & $-13.12 \mathrm{~dB}$ & $-9.84 \mathrm{~dB}$ \\
\hline Point 9 & $0.87 \mathrm{~m}$ & $-13.07 \mathrm{~dB}$ & $-9.81 \mathrm{~dB}$ \\
\hline \multicolumn{4}{|c|}{$x$-Direction } \\
\hline Point & 3-dB width & PSLR & ISLR \\
\hline Point 1 & $0.48 \mathrm{~m}$ & $-11.68 \mathrm{~dB}$ & $-9.67 \mathrm{~dB}$ \\
\hline Point 2 & $0.47 \mathrm{~m}$ & $-11.83 \mathrm{~dB}$ & $-9.49 \mathrm{~dB}$ \\
\hline Point 3 & $0.53 \mathrm{~m}$ & $-11.64 \mathrm{~dB}$ & $-9.57 \mathrm{~dB}$ \\
\hline Point 4 & $0.45 \mathrm{~m}$ & $-12.04 \mathrm{~dB}$ & $-9.48 \mathrm{~dB}$ \\
\hline Point 5 & $0.46 \mathrm{~m}$ & $-12.17 \mathrm{~dB}$ & $-9.37 \mathrm{~dB}$ \\
\hline Point 6 & $0.47 \mathrm{~m}$ & $-11.41 \mathrm{~dB}$ & $-9.60 \mathrm{~dB}$ \\
\hline Point 7 & $0.49 \mathrm{~m}$ & $-10.79 \mathrm{~dB}$ & $-9.43 \mathrm{~dB}$ \\
\hline Point 8 & $0.52 \mathrm{~m}$ & $-11.80 \mathrm{~dB}$ & $-9.40 \mathrm{~dB}$ \\
\hline Point 9 & $0.52 \mathrm{~m}$ & $-12.24 \mathrm{~dB}$ & $-9.59 \mathrm{~dB}$ \\
\hline
\end{tabular}

Table 4. The focusing quality of the point scatterers from the proposed algorithm.

\begin{tabular}{|c|c|c|c|}
\hline \multicolumn{4}{|c|}{$y$-Direction } \\
\hline Point & 3-dB width & PSLR & ISLR \\
\hline Point 1 & $0.86 \mathrm{~m}$ & $-13.11 \mathrm{~dB}$ & $-9.88 \mathrm{~dB}$ \\
\hline Point 2 & $0.85 \mathrm{~m}$ & $-12.99 \mathrm{~dB}$ & $-9.98 \mathrm{~dB}$ \\
\hline Point 3 & $0.86 \mathrm{~m}$ & $-13.09 \mathrm{~dB}$ & $-9.87 \mathrm{~dB}$ \\
\hline Point 4 & $0.85 \mathrm{~m}$ & $-13.11 \mathrm{~dB}$ & $-9.99 \mathrm{~dB}$ \\
\hline Point 5 & $0.85 \mathrm{~m}$ & $-13.10 \mathrm{~dB}$ & $-9.97 \mathrm{~dB}$ \\
\hline Point 6 & $0.86 \mathrm{~m}$ & $-12.96 \mathrm{~dB}$ & $-9.84 \mathrm{~dB}$ \\
\hline Point 7 & $0.86 \mathrm{~m}$ & $-13.01 \mathrm{~dB}$ & $-9.88 \mathrm{~dB}$ \\
\hline Point 8 & $0.87 \mathrm{~m}$ & $-13.02 \mathrm{~dB}$ & $-9.76 \mathrm{~dB}$ \\
\hline Point 9 & $0.87 \mathrm{~m}$ & $-13.13 \mathrm{~dB}$ & $-9.93 \mathrm{~dB}$ \\
\hline \multicolumn{4}{|c|}{$x$-Direction } \\
\hline Point & 3-dB width & PSLR & ISLR \\
\hline Point 1 & $0.46 \mathrm{~m}$ & $-12.91 \mathrm{~dB}$ & $-9.78 \mathrm{~dB}$ \\
\hline Point 2 & $0.48 \mathrm{~m}$ & $-13.03 \mathrm{~dB}$ & $-9.87 \mathrm{~dB}$ \\
\hline Point 3 & $0.51 \mathrm{~m}$ & $-12.91 \mathrm{~dB}$ & $-9.91 \mathrm{~dB}$ \\
\hline Point 4 & $0.43 \mathrm{~m}$ & $-13.03 \mathrm{~dB}$ & $-9.93 \mathrm{~dB}$ \\
\hline Point 5 & $0.43 \mathrm{~m}$ & $-13.02 \mathrm{~dB}$ & $-9.92 \mathrm{~dB}$ \\
\hline Point 6 & $0.46 \mathrm{~m}$ & $-12.99 \mathrm{~dB}$ & $-9.87 \mathrm{~dB}$ \\
\hline Point 7 & $0.48 \mathrm{~m}$ & $-12.91 \mathrm{~dB}$ & $-9.81 \mathrm{~dB}$ \\
\hline Point 8 & $0.50 \mathrm{~m}$ & $-13.00 \mathrm{~dB}$ & $-9.89 \mathrm{~dB}$ \\
\hline Point 9 & $0.51 \mathrm{~m}$ & $-12.89 \mathrm{~dB}$ & $-9.79 \mathrm{~dB}$ \\
\hline
\end{tabular}




\subsection{Raw Data Experiments for Airborne SAR}

To further evaluate the performance of the proposed algorithm, raw data experiments from a maneuvering highly-squint airborne SAR system are implemented by utilizing the proposed MOCO with CFBP algorithm. The airborne SAR is operating in a Ka band with a bandwidth of $200 \mathrm{MHz}$. The range center is about $7000 \mathrm{~m}$. The forward velocity of the airborne platform is about $60 \mathrm{~m} / \mathrm{s}$ and the downward velocity of the airborne platform is about $1.1 \mathrm{~m} / \mathrm{s}$. The squint angle is about 45 degrees. Because the on-board INS/GPS information is not accurate enough, data-driven MOCO is processed with CFBP. By using the proposed $\mathrm{MOCO}$, the estimated motion error of the airborne platform is shown in Figure 12. From Figure 12, it can be seen that the motion error exceeds one quarter of the scale of wavelength, which needs to be compensated in the CFBP process.

The focusing results are provided in Figure 13. The size of the final SAR image in terms of $X \times Y$ is $600 \mathrm{~m} \times 800 \mathrm{~m}$. Figure 13a is the result obtained by the CFBP algorithm without any $\mathrm{MOCO}$, and it can be seen that the image is degraded seriously with the image entropy of 11.203. Figure 13b is the results obtained by data-driven MOCO in CFBP, but the MOCO is not in a spectrum-compressed domain. From Figure 13b, it can be seen that obvious defocusing remains in the image with the image entropy of 10.751 . Figure $13 \mathrm{c}$ is the results obtained from the algorithm of [27]. Because the spatial-variant phase error cannot be accurately compensated, minor defocusing still remains in the final image with the entropy of 10.662. Figure 13d is the results obtained from the proposed algorithm in which the data-driven MOCO is processed in spectrum-compressed domain, also combined with the CFBP algorithm. From Figure 13d, it can be seen that the algorithm achieves the highest focusing quality with image entropy of 10.628 . Figure 14 compares the zoomed images. It is shown that the buildings as well as the roads can be clearly distinguished in the SAR image, which shows that the proposed MOCO algorithm can effectively compensate the unknown motion error to achieve better performance. Figure 15 compares the azimuth responses of two focused prominent scatterers. The azimuth resolution obtained from the algorithm of [27] is about $0.125 \mathrm{~m}$, while the azimuth resolution obtained from the proposed algorithm is about $0.110 \mathrm{~m}$. From the comparison, it can be seen that the proposed algorithm can achieve higher focusing quality, which validates the superior performance of the proposed algorithm.

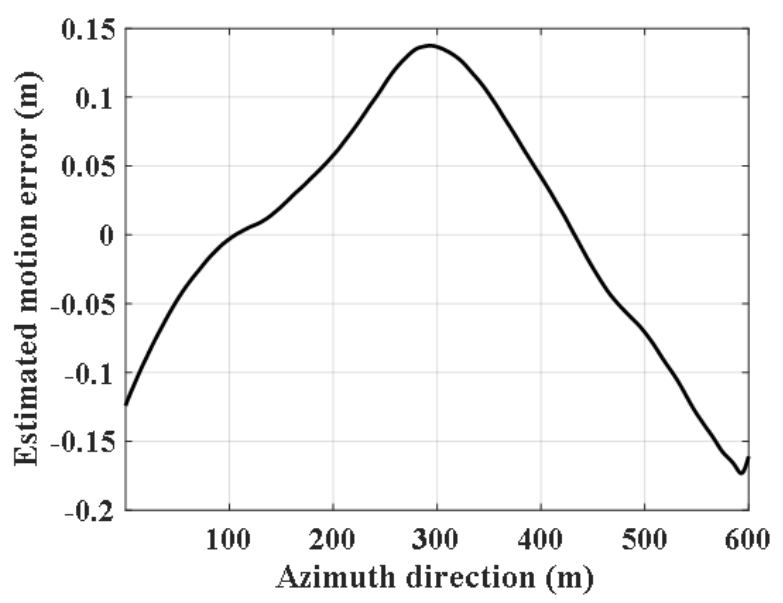

Figure 12. Motion error of airborne platform estimated from echoes. 


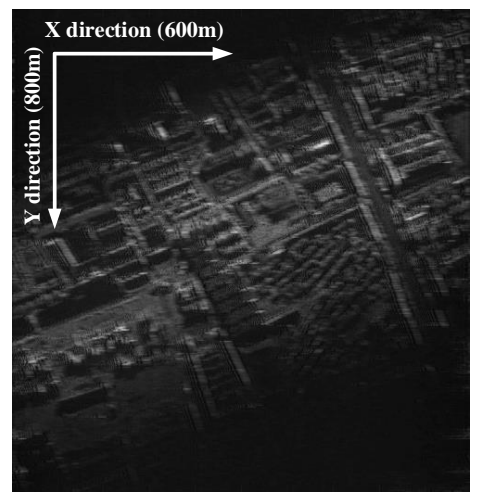

(a)

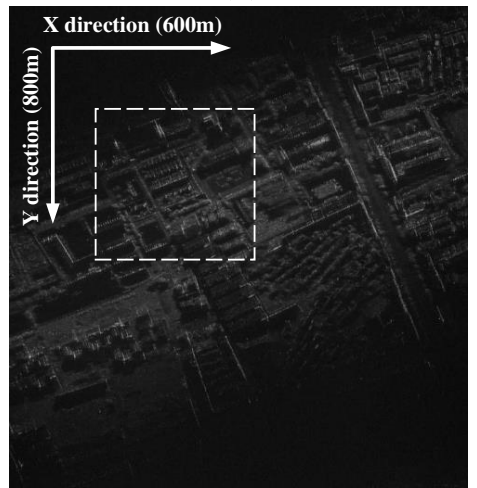

(c)

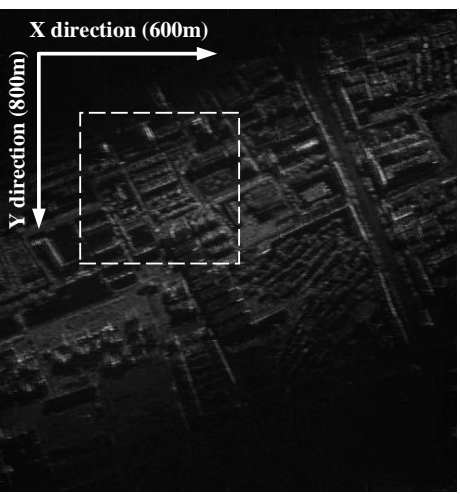

(b)

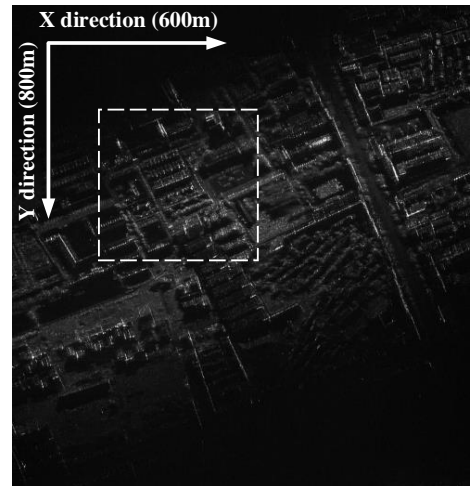

(d)

Figure 13. Experimental results from highly-squint airborne SAR. (a) Obtained results from CFBP algorithm without any MOCO; (b) obtained results from CFBP algorithm combined with data-driven MOCO but without spectrum compression; (c) obtained results from the algorithm of [27]; (d) obtained results from the proposed algorithm.

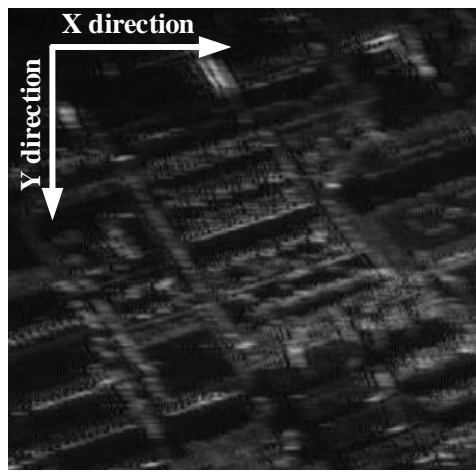

(a)

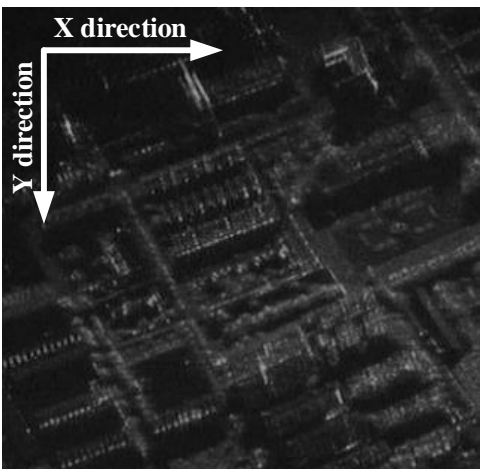

(b)

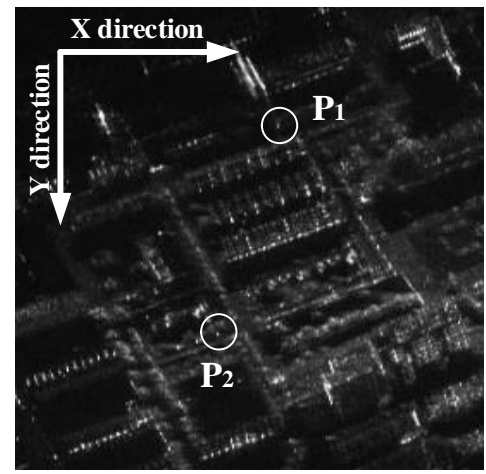

(c)

Figure 14. Zoomed images for comparison. (a) Obtained results from CFBP algorithm combined with data-driven MOCO but without spectrum compression; (b) obtained results from the algorithm of [27]; (c) obtained results from the proposed algorithm. 

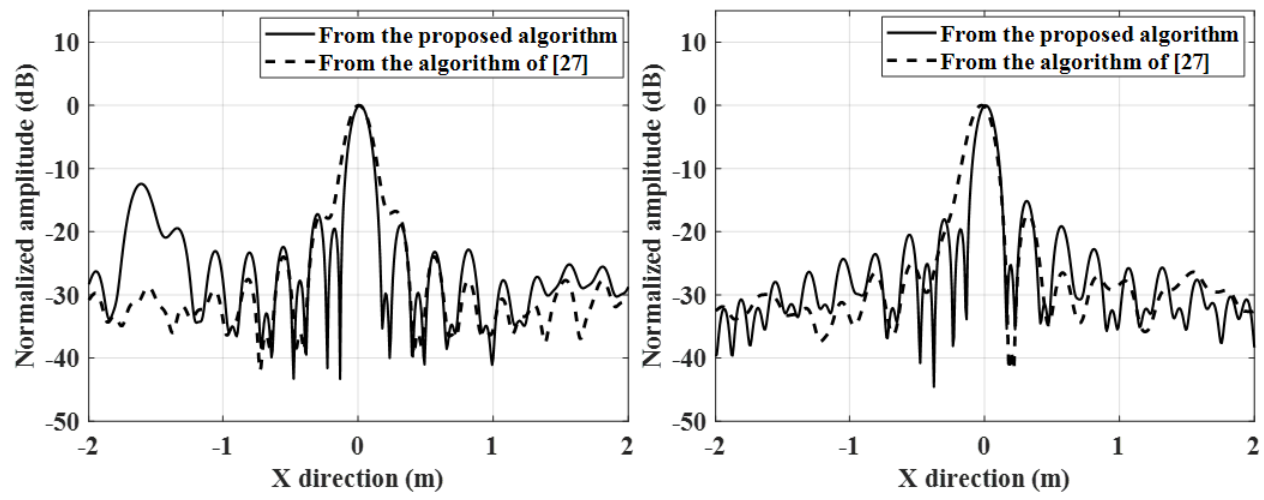

Figure 15. Azimuth responses of the prominent scatterers of $P_{1}$ and $P_{2}$. The dashed lines denote the results from the algorithm of [27], and the solid lines denote the results from the proposed algorithm.

\section{Conclusions}

In this paper, a new data-driven MOCO algorithm is developed under the CFBP framework for missile-borne SAR application. The SAR image from CFBP is transformed into a spectrum-compressed domain where the echoes from different scatterering points are aligned in the same spectrum range in a spectrum-compressed domain, and the phase error can be regarded as spatially invariant. With this inherent characteristic of spectrumcompressed domain, an effective data-driven $\mathrm{MOCO}$ algorithm is developed with CFBP algorithm for accurate error estimation and compensation. Results from simulations and raw data experiments are presented to validate the performance of the proposed algorithm.

Author Contributions: M.B. developed the proposed algorithm and wrote the manuscript. M.B. and S.Z. conducted the experiments and organized the manuscript. M.X. commented on the manuscript and made useful suggestions. All authors have read and agreed to the published version of the manuscript.

Funding: This work was supported in part by the National Key R \& D Program of China under Grant 2018YFC0810202, and in part by Fire and Rescue Department technology Program of MEM under Grant 2020XFZD05, and in part by the National Natural Science Foundation of China under Grant Nos. 61201284, 61801204, and 62061031, and in part by Shaanxi Innovative Talents Promotion Plan-Science and Technology Innovation Team under Grant No. 2019TD-002, and in part by the public foundation from the Key Laboratory of EMW Information, Fudan University, China, under Grant No. EMW201901.

Institutional Review Board Statement: Not applicable.

Informed Consent Statement: Not applicable.

Data Availability Statement: Not applicable.

Conflicts of Interest: The authors declare no conflict of interest.

\section{Abbreviations}

The following abbreviations are used in this manuscript:

LD linear dichroism

SAR synthetic aperture radar

FFBP fast factorized back-projection

CFBP Cartesian factorized back projection

MOCO motion compensation

FDA frequency-domain algorithm

TDA time-domain algorithm

RDA range-doppler algorithm 


$\begin{array}{ll}\text { CSA } & \text { chirp-scaling algorithm } \\ \text { BP } & \text { back-projection } \\ \text { INS/GPS } & \text { inertial navigation system/global positioning system } \\ \text { UAV } & \text { unmanned aerial vehicle } \\ \text { FT } & \text { Fourier transformation } \\ \text { SA } & \text { sub image } \\ \text { POSP } & \text { principle of stationary phase } \\ \text { PGA } & \text { phase gradient autofocusing } \\ \text { MDA } & \text { map-drift autofocusing } \\ \text { WPGA } & \text { weighted PGA } \\ \text { APC } & \text { antenna phase center } \\ \text { NsRCM } & \text { nonsystematic range cell migration } \\ \text { PRF } & \text { pulse repetition frequency } \\ \text { PSLR } & \text { peak sidelobe ratio } \\ \text { ISLR } & \text { integrated sidelobe ratio }\end{array}$

\section{References}

1. Zink, M.; Moreira, A.; Hajnsek, I.; Rizzoli, P.; Bachmann, M.; Kahle, R.; Fritz, T.; Huber, M.; Krieger, G.; Lachaise, M. TanDEM-X: 10 Years of Formation Flying Bistatic SAR Interferometry. IEEE J. Sel. Top. Appl. Earth Obs. Remote Sens. 2021, 14, $3546-3565$. [CrossRef]

2. Wan, J.; Tan, X.; Chen, Z.; Dong, L.; Liu, Q.; Zhou, Y.; Zhang, L. Refocusing of Ground Moving Targets with Doppler Ambiguity Using Keystone Transform and Modified Second-Order Keystone Transform for Synthetic Aperture Radar. Remote Sens. 2021, 2, 177. [CrossRef]

3. Freeman, A.; Zink, M.; Caro, E.; Moreira, A.; Veilleux, L.; Werner, M. The legacy of the SIR-C/X-SAR radar system: 25 years on. Remote Sens. Environ. 2019, 231, 111255. [CrossRef]

4. Luebeck, D.; Wimmer, C.; Moreira, L.F.; Alcântara, M.; Oré, G.; Góes, J.A.; Oliveira, L.P.; Teruel, B.; Bins, L.S.; Gabrielli, L.H Drone-borne differential SAR interferometry. Sensors 2020, 12, 778. [CrossRef]

5. Bie, B.; Sun, G.C.; Xia, X.G.; Xing, M.; Guo, L.; Bao, Z. High-speed maneuvering platforms squint beam-steering SAR imaging without subaperture. IEEE Trans. Geosci. Remote Sens. 2019, 57, 6974-6985. [CrossRef]

6. Li, G.; Ma, Y.; Xiong, X. Maneuvering platform high-squint SAR imaging method based on perturbation KT and subregion phase filtering. J. Appl. Remote Sens. 2021, 1, 016502.

7. Feng, D.; An, D.; Huang, X. An extended fast factorized back projection algorithm for missile-borne bistatic forward-looking SAR imaging. IEEE Trans. Aerosp. Electron. Syst. 2018, 54, 2724-2734. [CrossRef]

8. Tang, S.; Guo, P.; Zhang, L.; Lin, C. Modeling and Precise Processing for Spaceborne Transmitter/Missile-Borne Receiver SAR Signals. Remote Sens. 2019, 11, 346. [CrossRef]

9. Li, X.; Zhou, S.; Yang, L. A New Fast Factorized Back-Projection Algorithm with Reduced Topography Sensibility for Missile-Borne SAR Focusing with Diving Movement. Remote Sens. 2020, 16, 2616. [CrossRef]

10. Cumming, I.G.; Wong, F.H. Digital Signal Processing of Synthetic Aperture Radar Data: Algorithms and Implementation; Artech House: Norwood, MA, USA, 2004.

11. Chen, J.; Xing, M.; Sun, G.; Li, Z. A 2D space-variant motion estimation and compensation method for ultrahigh-resolution airborne stepped-frequency SAR with long integration time. IEEE Trans. Geosci. Remote Sens. 2017, 55, 6390-6401. [CrossRef]

12. Rodriguez-Cassola, M.; Prats, P.; Krieger, G.; Moreira, A. Efficient time-domain image formation with precise topography accommodation for general bistatic SAR configurations. IEEE Trans. Aerosp. Electron. Syst. 2011, 47, 2949-2966. [CrossRef]

13. Desai, M.D.; Jenkins, W.K. Convolution backprojection image reconstruction for spotlight mode synthetic aperture radar. IEEE Trans. Image Process. 1992, 1, 505-517. [CrossRef] [PubMed]

14. Zhang, Y.; Zhang, Q.; Zhang, Y.; Pei, J.; Huang, Y.; Yang, J. Fast split bregman based deconvolution algorithm for airborne radar imaging. Remote Sens. 2020, 12, 1747. [CrossRef]

15. Gaibel, A.; Boag, A. Backprojection Imaging of Moving Objects. IEEE Trans. Image Process. 2020. [CrossRef]

16. Lin, C.; Tang, S.; Zhang, L.; Guo, P. Focusing High-Resolution Airborne SAR with Topography Variations Using an Extended BPA Based on a Time/Frequency Rotation Principle. Remote Sens. 2018, 8, 1275. [CrossRef]

17. Zhang, L.; Li, H.L.; Qiao, Z.J.; Xing, M.D.; Bao, Z. Integrating autofocus techniques with fast factorized back-projection for high-resolution spotlight SAR imaging. IEEE Geosci. Remote Sens. Lett. 2013, 10, 1394-1398. [CrossRef]

18. Mao, X.; Ding, L.; Zhang, Y.; Zhan, R.; Li, S. Knowledge-Aided 2D Autofocus for Spotlight SAR Filtered Backprojection Imagery. IEEE Trans. Geosci. Remote Sens. 2019, 57, 9041-9058. [CrossRef]

19. Ulander, L.M.H.; Hellsten, H.; Stenstrom, G. Synthetic-aperture radar processing using fast factorized back-projection. IEEE Trans. Aerosp. Electron. Syst. 2003, 39, 760-776. [CrossRef]

20. Shippey, G.; Banks, S.; Pihl, J. SAS image reconstruction using fast polar back projection: Comparisons with fast factored back projection and Fourier-domain imaging. Eur. Ocean. 2005, 1, 96-101. 
21. Yang, Z.; Sun, G.-C.; Xing, M. A new fast back-projection algorithm using polar format algorithm. In Proceedings of the 2013 Asia-Pacific Conference on Synthetic Aperture Radar (APSAR), Tsukuba, Japan, 23-27 September 2013; Volume 1, pp. 373-376.

22. Vu, V.T.; Pettersson, M.I. Nyquist sampling requirements for polar grids in bistatic time-domain algorithms. IEEE Trans. Signal Process. 2014, 63, 457-465. [CrossRef]

23. Zhou, S.; Yang, L.; Zhao, L.; Wang, Y.; Zhou, H.; Chen, L.; Xing, M. A New Fast Factorized Back Projection Algorithm for Bistatic Forward-Looking SAR Imaging Based on Orthogonal Elliptical Polar Coordinate. IEEE J. Sel. Top. Appl. Earth Obs. Remote Sens. 2019, 12, 1508-1520. [CrossRef]

24. Dong, Q.; Sun, G.; Yang, Z.; Guo, L.; Xing, M. Cartesian factorized backprojection algorithm for high-resolution spotlight SAR imaging. IEEE Sens. J. 2017, 3, 1160-1168. [CrossRef]

25. Luo, Y.; Zhao, F.; Li, N.; Zhang, H. A modified cartesian factorized back-projection algorithm for highly squint spotlight synthetic aperture radar imaging. IEEE Geosci. Remote Sens. Lett. 2018, 6, 902-906. [CrossRef]

26. Chen, X.; Sun, G.; Xing, M.; Li, B.; Yang, J.; Bao, Z. Ground Cartesian Back-Projection Algorithm for High Squint Diving TOPS SAR Imaging. IEEE Trans. Geosci. Remote Sens. 2020. [CrossRef]

27. Luo, Y.; Zhao, F.; Li, N.; Zhang, H. An autofocus cartesian factorized backprojection algorithm for spotlight synthetic aperture radar imaging. IEEE Geosci. Remote Sens. Lett. 2018, 8, 1244-1248. [CrossRef]

28. Xing, M.; Jiang, X.; Wu, R.; Zhou, F.; Bao, Z. Motion compensation for UAV SAR based on raw radar data. IEEE Trans. Geosci. Remote Sens. 2009, 8, 2870-2883. [CrossRef]

29. Zhang, L.; Qiao, Z.; Xing, M.; Yang, L.; Bao, Z. A robust motion compensation approach for UAV SAR imagery. IEEE Trans. Geosci. Remote Sens. 2012, 8, 3202-3218. [CrossRef]

30. Pu, W. Deep SAR Imaging and Motion Compensation. IEEE Trans. Image Process. 2021, 30, 2232-2247. [CrossRef]

31. Zhou, S.; Yang, L.; Zhao, L.; Bi, G. Quasi-polar-based FFBP algorithm for miniature UAV SAR imaging without navigational data. IEEE Trans. Geosci. Remote Sens. 2017, 55, 7053-7065. [CrossRef]

32. $\mathrm{Pu}, \mathrm{W} . ; \mathrm{Wu}, \mathrm{J} . ;$ Huang, Y.; Yang, J.; Yang, H. Fast Factorized Backprojection Imaging Algorithm Integrated With Motion Trajectory Estimation for Bistatic Forward-Looking SAR. IEEE J. Sel. Top. Appl. Earth Obs. Remote Sens. 2019, 12, 3949-3965. [CrossRef]

33. Yang, L.; Li, P.; Zhang, S.; Zhao, L.; Zhou, S.; Xing, M. Cooperative Multitask Learning for Sparsity-Driven SAR Imagery and Nonsystematic Error Autocalibration. IEEE Trans. Geosci. Remote Sens. 2020, 58, 5132-5147. [CrossRef]

34. Wahl, D.; Eichel, P.H.; Ghiglia, D.C.; Jakowatz, C.V. Phase gradient autofocus-a robust tool for high resolution SAR phase correction. IEEE Trans. Aerosp. Electron. Syst. 1994, 3, 827-835. [CrossRef]

35. Ye, W.; Yeo, T.; Bao, Z. Weighted least-squares estimation of phase errors for SAR/ISAR autofocus. IEEE Trans. Geosci. Remote Sens. 1999, 5, 2487-2494. [CrossRef]

36. Bao, M.; Zhou, S.; Yang, L.; Xing, M.; Zhao, L. Data-driven Motion Compensation for Airborne Bistatic SAR Imagery under Fast Factorized Back Projection Framework. IEEE J. Sel. Top. Appl. Earth Obs. Remote Sens. 2021, 14, 1728-1740. [CrossRef] 